\title{
EXPERIENCIA DE LABORATORIO NO ESTRUCTURADO EN LA UNIVERSIDAD
}

herrera CABello, f, y Corullon paredes, a.

Dpto. de Física, Universidad de La Laguna (Tenerife)

\section{SUMMARY}

This paper is an introduction to the unstructured laboratory, mentioned by Robinson (1979) and Spears-Zollman (1977) for university level. This experience was performed at the Optical Laboratory by the third year physics course students at La Laguna University.

\section{INTRODUCCION}

Es bastante común que los alumnos de las Facultades de Ciencias de nuestro país terminen con una pobre formación experimental en Física y bastante desligada de la realidad científica del momento. Se suele decir que las causas son la escasez de medios económicos y la infravaloración del Laboratorio dentro de la actividad docente.

Nosotros mostramos aquí un ejemplo de experiencia de laboratorio, montada con material convencional, no demasiado caro, en la que hemos intentado poner a los alumnos en contacto con algunos aspectos de la investigación actual, además de mostrarles que la Física es algo vivo y existe una relación entre cada una de sus ramas.

Con motivo del proceso de montaje del laboratorio de la asignatura de Optica, por primera vez en la Universidad de La Laguna, se pensó darle un enfoque totalmente distinto al de los ya existentes de Electromagnetismo o Física General (montados en la forma clásica), tratando que el alumno pusiera más de su parte en cada experiencia. Por ello, como última práctica decidimos montar la experiencia que se describe en este trabajo aprovechando, además, que el nivel de conocimientos de Optica y Estructura de la Materia de los alumnos, cercano ya el final del curso, nos permitían tocar los puntos principales que se tratan en la misma. Por otro lado, nuestros alumnos serán la primera promoción de Físicos que se formarán totalmente en La Laguna, por lo que están acostumbrados desde $1^{\circ}$ a ser un poco el blanco de las innovaciones de los profesores. Este detalle nos hizo pensar que se evitaría así el efecto Hawtorn (el resultado del experimento cambia, cuando el alumno sabe que es objeto del mismo). A modo de curiosidad, diremos que estas personas, desde que realizaron en $1^{\circ}$ las prácticas de laboratorio de Física General, no habían vuelto a tomar contacto con la Física experimental hasta $3^{\circ}$, curso en el que se en- contraron con las prácticas de Optica y Electromagnetismo.

\section{LA EXPERIENCIA}

\subsection{Objetivos}

Para organizar la experiencia, se tomó como referencia la taxonomía de Klopfer (1975) y los objetivos seleccionados de la misma son los que se citan a continuacion:

Categoría A: $1,2,3,5,6,7,8$ y 10

(Conocimiento y comprensión)

Categoria B: 1, 2, 3, 4 y 5

(Observación y medida)

Categoria C: $1,2,3$ y 4

(Descubrimiento de un problema y búsqueda de la forma de resolverlo)

Categoría D: $1,3,5$ y 6

(Interpretación de datos y formulación de generalizaciones)

Categoria F: 1 y 3

(Aplicación de conocimientos y métodos científicos)

Categoría G: 1 y 2

(Destrezas manuales)

Categoría $\mathrm{H}: 1,2,4,5$ y 6

(Actitudes e intereses)

Categoría I: 3,4 y 5

(Orientación)

\subsection{Diseño del experimento}

Nuestros alumnos se agruparon, por afinidad, en equipos de 4 para la realización de la experiencia. Esto hubo que hacerlo por razones de espacio y tiempo, pero también nos pareció conveniente que pudieran cambiar 
impresiones mientras trabajaban. Cada miembro del equipo intervendria en la toma de medidas, llevaría un cuaderno de laboratorio y presentaria al final un informe, similar al de los presentados por los científicos en revistas especializadas. (En esto estamos de acuerdo con autores como Robinson (1979)). En las otras prácticas de Optica, se limitaban a tomar notas y a presentar un resumen, que no se les pediría hasta el final de las mismas.

La evaluación de la experiencia se hizo respecto a criterio (Gardener), asignando el valor 1 ó 0 si el objeti-. vo estudiado era alcanzado o no por el alumno. Dicha Evaluación se realizó a partir de los informes, cuadernos de laboratorio, observaciones del profesor y un examen tipo test.

\subsection{Desarrollo de la experiencia}

En la figura 1 se muestra un esquema del montaje bá sico utilizado y en el Apéndice II incluimos una des. cripción del material que aparece en ella.

Desde el punto de vista de la asignatura, los objetivos que se querían conseguir eran:

A) Familiarización del alumno con el LASER mediante: a) Comprensión del fundamento y la obtención de la radiación LASER b) Estudio de las caracteristicas del haz utilizado.

B) Estudiar las diversas formas de actuar sobre un haz plano-polarizado utilizando la acción de campos eléctricos y magnéticos, que al actuar sobre ciertos materiales inducen en ellos propiedades anisótropas las cuales se ponen de manifiesto al ser atravesadas por un haz de luz monocromático linealmente polarizado. Esto se hizo: a) Obteniendo un haz elipticamente polariza do a partir de uno plano-polarizado. (Usando el ef́ecto electroóptico transversal en una cerámica ferroeléctrica y midiendo su constante electroóptica). b) Rotan- do el plano de polarización del haz. (Usando la rotación Faraday y midiendo la constante de Verdet para un vidrio Flint).

C) Destreza en el manejo de un sistema básico de medida.

D) Comprobar la interrelación entre las diferentes áreas de la Física como son: a) La Física de Materiales, porque en ella se estudia el comportamiento de los mismos. b) El Electromagnetismo, porque permite el cálculo aproximado del campo magnético usado en la rotación Faraday. c) La Electrónica, porque nos per* mite medir la intensidad de haz.

La organización de la experiencia fue como sigue:

En la Primera Sesion, de duración aproximada de 3 horas, se les indicaron las normas generales, como la necesidad de llevar un cuaderno de laboratorio y confeccionar un informe, dándoles fotocopias de un articulo cientifíco extraido de la revista Journal of NonCrystalline Solids (1971), para que les sirviera de mo. delo. A continuación una charla sobre el LASER ex. plicándoles la historia de su descubrimiento, fundamentos y aplicaciones más importantes; asimismo se les proporcionó una fotocopia de un catálogo comercial sobre el equipo, indicándoles el significado de sus especificaciones, mostrándoles además el montaje básico que utilizarian asi como otra fotocopia con las características del fotodetector. Para que no fueran con ideas preconcebidas, sólo se les dijo lo que iban a hacer en la Segunda Sesión. En esta, cuya duración fue de aproximadamente 2 horas, estudiaron las caracteristicas del haz LASER que utilizarían, el cual tenía cierto defecto cuyas causas deberian determinar. También realizaron unas cuantas medidas encaminadas al estudio de la sensibilidad del sistema de detección, familiarizán. dose de este modo con las dificultades del manejo de la instrumentación y del montaje experimental (los aparatos eran de diferente procedencia y todo el conjunto

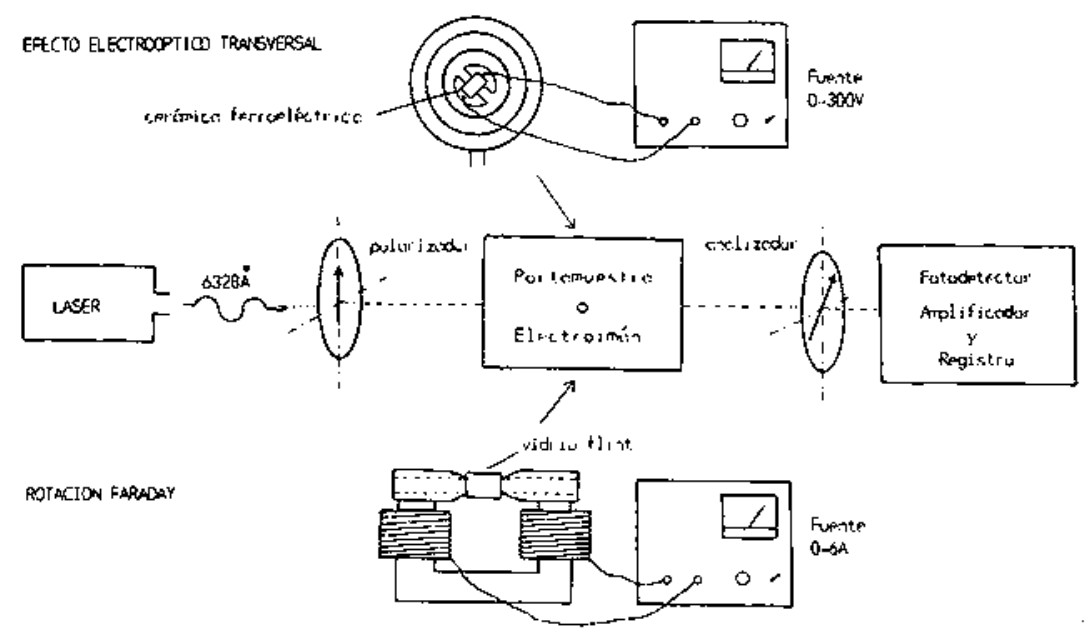


pretendia ser una muestra de cómo un investigador empieza a tomar contacto con el fenómeno a investigar, improvisando sobre la marcha la forma de estudiar las diferentes variables que intervienen). En esta sesión trabajaron basándose únicamente en unas ideas generales que se les dieron al principio de la misma. Es decir, no hubo ningún guión y tuvieron que poner bastante de su ingenio.

En la Tercera sesión, que duró unas 3 horas, utilizaron separatas en las que aparecían los conceptos teori. cos más importantes que les permitirian comprender las observaciones y medidas que deberian realizar en la siguiente sesión. (Borrelli y Layton, 1971), (Elliot, 1966), (Kittel, 1981) y (Layton y Herczog, 1967).

A su vez, se les habló de las características (fabrica* ción y propiedades dieléctricas) del material ferroeléc. trico que iban a utilizar: una muestra de cerámica transparente del tipo PLZT, amablemente cedida por el Instituto de Física de Materiales del C.E.N.F.A. "Torres Quevedo». En cuanto a la rotación Faraday, se les explicó también la que caracteriza a los ferrimagnéticos, comentándoles en la última sesión sus aplicaciones en las guias de ondas.

En la Cuarta Sesión, de duración aproximada 5 horas, tuvieron que hacer medidas que exigían soltura en la manipulación de los aparatos, observaron su compor* tamiento y comprobaron la validez de una aproximación teórica y la veracidad de algunas leyes. Para ello todos dispusieron de un guión (que adjuntamos en el Apéndice I), que les obligaba a seguir un orden de trabajo, parcialmente perfilado, intercalando cuestiones que debian responder al profesor de prácticas antes de poder continuar con la experiencia; a su vez se les pedia que sugiriesen los métodos de medida y las condiciones óptimas para su realización.

Por tultimo, en la Quinta Sesión, tuvo lugar un examen tipo test sobre los conceptos teóricos y técnicas utilizadas en la experiencia; comentándoles a continuación. la influencia y aplicaciones presentes y futuras en el campo de las comunicaciones de los fenómenos observados.

\section{RESULTADOS Y DISCUSION}

Antes de exponer los resultados, cabe señalar que la práctica, dada su duración y periodo en que fue realin zada (plenos exámenes) fue de carácter voluntario. Llevados por la curiosidad, participaron la totalidad de los alumnos, aunque a la hora de presentar los trabajos relacionados con la misma, únicamente lo hicieron 8 de los 15 alumnos que integraban el curso. Podemos afirmar que aquellos que nos entregaron sus trabajos, han denotado un gran interés por la asignatura $y$, por ello, hemos supuesto que han alcanzado con creces los objetivos de las categorias I y $\mathrm{H}$, a excepción de la $\mathrm{H}-2$, que se evaluó en virtud de su forma de trabajar duran. te la experiencia.
Los resultados obtenidos aparecen en la tabla 1 .

Tabla I

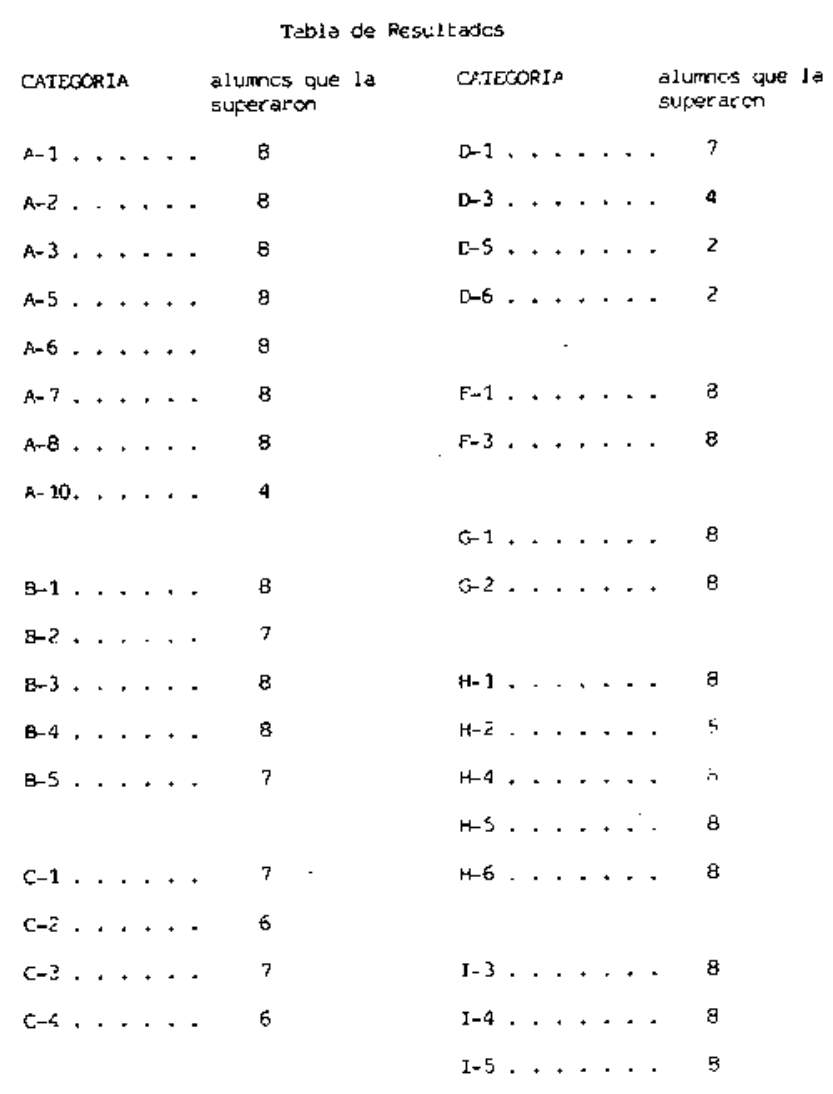

Vemos que las categorias en que el nivel ha sido más bajo son la $\mathrm{D}$ y la $\mathrm{C}$. En la $\mathrm{C}$ nos centramos en la eva* luación de cómo el alumno era capaz de explicar las anomalías observadas en el LASER mediante las medidas realizadas en la $1^{\text {a }}$ parte experimental, en las que únicamente manejaban conceptos ya estudiados en el curso de Optica. En la categoría D, incluimos también los datos experimentales observados en la $2^{a}$ parte experimental, donde ya tenian que hacer uso de conceptos más nuevos; de ahí que se note la diferencia de una categoria a otra.

\section{CONCLUSIONES}

Aun teniendo en cuenta que los resultados anteriores no son muy representativos, entre otras cosas por el pequeño número de alumnos que participaron, creemos que no deja de ser interesante el que este grupo de personas, que apenas habían trabajado en un laboratorio y cuyo único contacto con la Física experimen. tal era a través de práctica «con recetario», hayan sido capaces de:

- Realizar medidas experimentales bastante aceptables, usando un montaje experimental de cierta dificultad de manejo y poca estabilidad. 
- Formular hipótesis y aprender a contrastarlas con los datos experimentales.

- Proponer métodos de medida adecuados a los medios de que disponían.

$\rightarrow$ Hacer un balance del trabajo realizado con un criterio bastante próximo al de una persona que se mue-

\section{APENDICE I}

\section{Apartado $1^{\circ}$}

Ej fin de esta experiencia es calcular el valor de ja constante electroóptica del material ferroelèctrico a la temp. ambiente. Habrá de tenerse en cuenta que la relación existente entre la birrefringencia inducida por el campo $\mathrm{E}$ y el valor del mismo, para este tipo de ma. teriales es $\triangle \mathrm{n}=\mathrm{B} \lambda \mathrm{E}^{2}$, siendo $\lambda$ ia longitud de onda del haz utiiizado y $\mathrm{B}$ la constante electrooptica de! material.

Resolver el siguiente cuestionario:

1) Forma de colocar la muestra con el campo aplicado, para obtenet la mejor respuesta a la salida del analizador.

2) Estudiar la respuesta del material utilizado para los diferentes valores del carnpo aplicado y trazar el gráfico correspondiente.

3) Método para medir experimentaimente el valor de la birrefringencia con los medios que tiene a su disposición. (En el laboratorio no teniamos lámina de cuarto de onda o un compensador óptico, por eso los alumnos tenian que sugerir y usar después los paráme tros de Stokes estudiados eri el curso de Optica, asi, debian saber que el haz a la \$alida de la lámina viene caracterizado por el vector de Stokes y presenta una diferencia de fase $S$

$$
S=\frac{2}{\lambda} \text { n.d donde } \lambda_{\mathrm{d}}=\text { longitud de onda en el vacio }
$$

deberà ser antalizado midiendo su intensidad al rotar el analizador).

4) Observar si existe o no bitrefringencia en ausencia de campo aplicado y, de ser asi, dar una explicación del fenómeno.

5) Calcular el valor de la constante electroóptica B a la temp. ambiente, explicando como se ha obtenido y el porqué.

\section{Apartado $2^{\circ}$}

Resolver el siguiente cuestionario:

1) Estudiar si la respuesta del detector es la adecuada para el campo de señales detectadas.

2) Determinar el valor del campo magnético para la máxima intensidad de corriente de la fuente y, por tanto, máxina intensidad en el detector. Para ello ha de tenet en cuenta que la constante de Verdet para el vidrio Flint a $18^{\circ} \mathrm{C}$ y a la longitud de onda del sodio es $V_{\mathrm{Na}_{2}}=3,17 \times 10^{\circ} \mathrm{si}$ se mide el ángulo en minutos de arco, el

\section{REFERENCIAS BIBLIOGRAFICAS}

BORRELLI, N.F. y LAYTON, M.M., 1971, Dielectric and Optical Properties of Transparent Ferroelectric Glass. Ceramic Systems, Journal of Non Crystalline Solids, $\mathrm{n}^{\circ}$ 6. pp. 197-212.

ELLIOT, R.S., 1966, Ferrimagnetism, Electromagnetics. (Mc Graw Hill: USA).

GARDENER, D.J., Journal of Higher Educ., 48(5), pp. $571-593$.

KITTEL, Ch., 1981, Cristales Ferroelécricos, Introducción a la Física del Estado Solido, (Edit. Reverté: Barcelona).

KLOPFER, J.W., 1975, La evaluación del aprendizaje en ve en un círculo científico.

Para terminar queremos decir que, con esta experiencia, hemos intentado empezar a trabajar en el campo de la Didáctica, dentro de la ensertanza universitaria. Agradecemos su colaboración a $\mathrm{M}^{2}$ del Carmen Pérez de Landazabal y a Basilio Jiménez del C.E.N.F.A. «Torres Quevedo».

campo magnético en teslas y la longitud en metros y que el valor del ángulo de rotación obtenido experimentalmente es de $3,9^{\circ}$ para una intensidad de corriente en el electroimán de 5,2A cuando se opera en la linea de $5893 \mathrm{~A}$ (del sodio). Se calcula así el valor del campo mediante $\theta=$ V.1.B.

3) Conociendo el valor del campo, determinar la constante de Verdet para la longitud de onda del LASER utilizado (Debian hacer uso de la expresión anterior: $\theta=$ V.I.B. siendo $\theta$ el ángulo de rotación, $V \mathrm{l}_{a}$ cte. de Verdet, $l$ el camino óptico y $B$ el campo aplicado).

4) Aplicando el valor del campo hallado en 2), comprueben la bondad de la aproximación: $\mathrm{B}=\frac{\mu_{0} \mathrm{~N} \text {.I }}{\mathrm{L}}$

siendo $\mu$ la permeabilidad en el vacío, $N$ el $n^{\circ}$ de espiras del electroimán, $L$ la longitud del gap (separación entre piezas polares) e $I$ la intensidad que recorre las espiras.

Esta aproximación fue tornada del capítulo 9 del texto de Lorrain, P. y Corson, D.R., 1972 Campos Magnéticos que estaba a su disposición en el laboratorio.

5) Hallar el ángulo de rotación Faraday para diferentes valores del campo aplicado.

\section{APENDICE II}

1.- Una fuente de luz, que es un LASER de gas $\mathrm{He}-\mathrm{Ne}$ de $1 \mathrm{~mW}$ de potencia y una longitud de onda de 6328A.

2.- Un dispositivo óptico que para el análisis de:

a) EI Efecto Electroóptico Transversal consta de

1 polarizador y 1 analizador

1 fuente de c.c. de $300 \mathrm{~V}$

1 portamuestra «ad hoc» donde se coloca una cerámica ferroeléctrica transparente de $0,2 \mathrm{~mm}$ de espesor, en la cual se habian impreso 2 electrodos utilizando una disolución de plata dejando una separación entre ellos de $1 \mathrm{~mm}$.

b) La Rotación Faraday comprende

1 analizador

1 electroiman compuesto de: I núcleo en forma de $\mathrm{U}, 2$ bobinas de 1000 espiras y un par de piezas polares perforadas

1 fuente de c.c. variable de $28 \mathrm{~V}-6 \mathrm{~A}$

3.- Un sistema de detección y registro formado por un fototransistor y un amplificador operacional cuya salida estaba conectada a un polímetro para medir la señal.

Ciencia, Cap. XVII de Evaluación del aprendizaje. Editado por Bloorn, Hasting y Madaus (Troquel: B. Aires).

LAYTON, M.M. y HERZOG, A., 1967, Nucleation and Crystallization of $\mathrm{NaNbO}_{3}$ from Glasses in the $\mathrm{Na}_{2} \mathrm{O}$ $\mathrm{Nb}, \mathrm{O}_{5}-\mathrm{SiO}$, System, Journal of American Ceramic Society, Vol. $50, \mathrm{~N}^{\circ} 7$, pp. $369-375$

LORRAIN, P. y CORSON, D.R., 1972, Circuito magnético con entrehierro de aire, Campos Magnéticos (III). (Selecciones Científicas: Madrid).

ROBINSON, M.C., 1979, Am, J. Phys. 47(10).

SPEARS, J. y ZOLLMAN, D., 1977, Journal of Research in Science Teaching, Vol. 14, $\mathrm{N}^{\circ} 1$, pp. 33-38. 